\section{A spin flipper for reversal of polarisation in a thermal atomic beam}

\author{
W Schröder and G Baum \\ Universität Bielefeld, Fakultät für Physik, D-4800 Bielefeld, \\ Federal Republic of Germany
}

Received 20 May 1981, in final form 26 July 1981

\begin{abstract}
By using the method of diabatic passage we developed a spin flipper which allows a rapid and convenient reversal of the atomic spins. The spin flipper was tested with a polarised lithium atomic beam by analysing the atomic beam polarisation with the help of a second spin-flip region and a sextupole magnet. The reversal efficiency was measured to be 0.98 which is in agreement with our calculation. The flipper is especially useful in low-energy electron-atom crossed-beam collision studies. Our experimental arrangement also allows a precise determination of the atomic beam polarisation.
\end{abstract}

\section{Introduction}

In some experiments with polarised atomic beams it is necessary to reverse frequently the direction of the atomic beam polarisation. Ideally this should be done without introducing changes in other experimental parameters. This task, however, is often difficult to accomplish. If, for instance, the atomic beam is polarised by optical pumping with circularly polarised light, reversal of the polarisation can be achieved by altering the sense of the light polarisation. In this case the reversal can easily lead to polarisation dependent atomic beam trajectories (Baum et al 1981). If, on the other hand, the atomic beam is polarised by passage through a sextupole magnet, which always produces the same sign of polarisation $\left(m_{s}=+1 / 2\right)$, one needs to reverse a magnetic field in the interaction region. In an electron-atom scattering experiment this method of polarisation reversal will affect the electron trajectories.

It has been known for a long time (Majorana 1932, Frisch and Segré 1933) that passage of spin-oriented atoms through weak magnetic fields near a zero-field point will give rise to nonadiabatic behaviour and changes of quantum states. In producing and transporting spin-oriented beams these spin-flip processes (Majerana transitions) are usually avoided by using magnetic guiding fields. With appropriately shaped magnetic fields, however, nonadiabatic transitions, which are also called diabatic transitions, can be exploited for effective spin reversal.

The condition for the occurrence of a spin flip is that the change of field direction seen by the atoms is fast compared to the Larmor precession frequency. This condition can be met in a spin flipper with weak and opposing longitudinal magnetic fields and a rapid crossing of zero in between. In this case, the atomic spins reverse their orientation with respect to the local magnetic field (diabatic mode of the spin fipper) but do not change their direction in space. For setting the spin flipper to the adiabatic mode a transverse magnetic field (about $0.1 \mathrm{mT}$ ) is applied at the field reversal region. This field has to be strong compared to the weak and opposing fields near the zero crossing. In the combined fields the atomic spins now precess rapidly and follow the local magnetic field direction adiabatically, turning through $180^{\circ}$ in space. The addition of the transverse field at the flip region, when going from the diabatic to the adiabatic mode, has a negligible influence on atomic beam intensity, atomic beam profile and magnetic field in the collision region. Our spin flipper is presently employed in an experiment on the spin-dependent interaction of polarised electrons with polarised lithium atoms. Here an asymmetry $A$ in the collision rate $N$ for parallel $(\uparrow \uparrow)$ and antiparallel $(\downarrow \uparrow)$ spin configurations is studied with $A=(N \uparrow \downarrow-N \uparrow \uparrow) /(N \uparrow \downarrow+N \uparrow \uparrow)$. As a check, null asymmetries, such as $(N \uparrow \downarrow-N \downarrow) /(N \uparrow \downarrow+N \downarrow)$, were measured and found to be consistent with zero to within \pm 0.001 , showing that the spin flipper is free of systematic effects on that level.

The diabatic reversal method is often used in nuclear physics experiments with polarised particles. There the diabatic conditions, outlined above, can be much more easily met than for thermal beams of paramagnetic atoms. Either the magnetic moment of the particles is small, as in the case of neutrons, or the particle velocity is high (about 100 times thermal velocity), as in the case of Lamb-shift hydrogen or deuterium sources. A spin flipper for neutrons has been described by Liaud et al (1975). Diabatic reversal techniques for Lamb-shift sources have been reviewed by Donally (1970) and McKibben (1980). Hight et al $(1977,1978)$ studied diabatic transition probabilities in a thermal atomic beam, theoretically as well as experimentally. Our work, reported on here, utilises the same principles as described by those authors, but emphasises the development of a spin-flipping device useful for efficient polarisation reversal.

\section{Spin dynamics}

The angular momentum $\boldsymbol{F}$ of an atom precesses with the Larmor frequency $\omega_{F}(t)$ in a magnetic field $B(t)$,

$$
\omega_{\mathrm{F}}(t)=g_{\mathrm{F}}\left(\mu_{\mathrm{B}} / \hbar\right) B(t) .
$$

Atoms propagating with velocity $v$ in a static non-uniform magnetic field see a time-varying magnetic field, $B(t)$. In low magnetic fields, where hyperfine coupling occurs, an atomic state can be described by

$$
\psi(t)=\sum_{F, m} A_{F, m}(t)|F, m\rangle .
$$

We use the diabatic representation, in which the eigenstates of the atoms are defined with respect to the atomic beam axis $(z$ axis). Note that in this representation $m$ refers to the projection of $\boldsymbol{F}$ along the $z$ axis. The time behaviour of the amplitude vector $A(t)$ is given by the equation of motion

$$
\mathrm{d} A(t) / \mathrm{d} t=-(\mathrm{i} / \hbar) \omega_{\mathrm{F}}(t)\left[\cos \theta(t) \hat{j}_{z}+\sin \theta(t) \hat{j}_{x}\right] A(t),
$$

where $\hat{j}_{x}$ and $\hat{j}_{z}$ are angular momentum operators and $\theta(t)$ is the polar angle between the local field direction and the $z$ axis.

In order to simplify equation (3) one can define a new amplitude $a_{F, m}(t)$ by

$$
a_{F, m}(t)=A_{F, m}(t) \exp \left(-\mathrm{i} m \int_{0}^{t} \omega_{F}\left(t^{\prime}\right) \cos \theta\left(t^{\prime}\right) \mathrm{d} t^{\prime}\right)
$$

which then leads to the equation of spin motion in the interaction picture:

$$
\begin{aligned}
\mathrm{d} a(t) / \mathrm{d} t=-(\mathrm{i} / \hbar) \omega_{\mathbf{F}}(t) \sin \theta(t)\left\langle F, m \pm 1\left|\hat{j}_{x}\right| F, m\right\rangle \\
\quad \times a(t) \exp \left( \pm \mathrm{i} \int_{0}^{t} \omega_{\mathbf{F}}\left(t^{\prime}\right) \cos \theta\left(t^{\prime}\right) \mathrm{d} t^{\prime}\right) .
\end{aligned}
$$

Under the assumption that the weak and opposing $B$ fields near the zero crossing are rotationally symmetric around the $z$ axis one has $\sin \theta(t)=0$ on axis (in the diabatic mode of the spin flipper). Equation (5) then shows for atoms propagating on axis that no change of the atomic eigenstates occurs. Atoms propagating off-axis at a radial distance $r$ through the zerocrossing region will see a magnetic field component in the radial 
direction ( $\sin \theta(t) \neq 0$ ), a consequence of the relation $\operatorname{div} \boldsymbol{B}=0$. According to equation (5) this can give rise to nonzero transition probabilities between eigenstates. No change of eigenstates means a change of the atomic spin directions with regard to the magnetic field and the occurrence of spin flips. Therefore transition probabilities between eigenstates have to be kept small for well-working flippers.

Transitions only occur between adjacent states within a hyperfine level. For low transition rates, as is the case for an efficient spin flipper, only the states adjacent to the one initially prepared will acquire a small, non-negligible population. It therefore suffices to calculate the transition probability $p_{F, m, m^{\prime}}$ between neighbouring states

$$
p_{F, m, m^{\prime}}=|I(r, v)|^{2} \hbar^{-2}\left|\left\langle F, m^{\prime}\left|\hat{j}_{x}\right| F, m\right\rangle\right|^{2}
$$

with

$$
\begin{aligned}
& I(r, v)=\mathrm{i} \int_{0}^{t} \omega_{\mathrm{F}}\left(t^{\prime}\right) \sin \theta\left(t^{\prime}\right) \\
& \quad \times \exp \left( \pm \mathrm{i} \int_{0}^{t^{\prime}} \omega_{\mathrm{F}}\left(t^{\prime \prime}\right) \cos \theta\left(t^{\prime \prime}\right) \mathrm{d} t^{\prime \prime}\right) \mathrm{d} t^{\prime}
\end{aligned}
$$

The last two equations were used for the numerical calculations presented in $\S 5$.

\section{Spin flipper design}

For adiabatic transport into and out of the low-field reversal region of the flipper we chose a current distribution which is aimed to approximate the following $B_{z}(z)$ dependence:

$$
\left|B_{z}\right|=2 \pi \hbar v\left(g_{\mathrm{F}} \mu_{\mathrm{B}} \ln \chi\right)^{-1}\left|\left(z-z_{\mathrm{E}}\right)\right|^{-1}
$$

where $z_{\mathrm{E}}\left(-z_{\mathrm{E}}\right)$ is the axial coordinate of the flipper exit (entrance). At both ends of the flipper the field $B_{z}$ deviates from equation (8), and merges into an external guiding field of about $1 \mathrm{mT}$. For the field shape of equation (8), the relative change in field strength along an axial distance traversed in one Larmor cycle is constant and is denoted by $\chi$. We chose $\chi=1.3$ as a compromise between reasonable flipper length $L$ and tolerable depolarisation in the adiabatic transport regions. In the midpoint of the flipper, between the opposing adiabatic transport fields, one has a nearly linear change of field strength (see figure 1) over an axial distance $d$ from $-B_{\mathrm{M}}$ to $+B_{\mathrm{M}}$. The value of $B_{\mathrm{M}}$ follows from equation (8) by taking $\left|z-z_{\mathrm{E}}\right|=L / 2$, in our case $B_{\mathrm{M}}=5 \mu \mathrm{T}$.

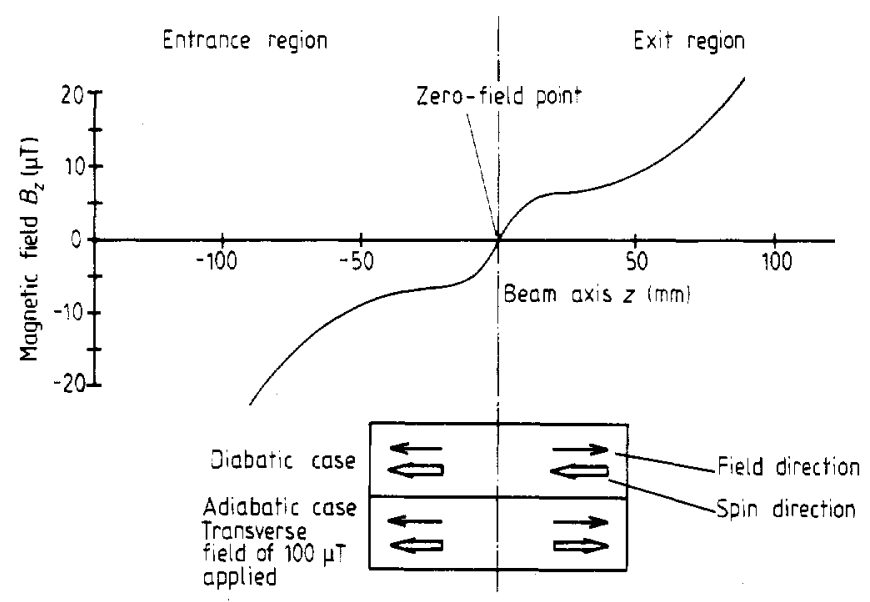

Figure 1. Magnetic field strength $B_{z}$ on the axis of the spin flipper. The arrows indicate the relative orientation of the magnetic field and spin direction for the two operational modes.
The unwanted depolarising transitions which occur in the reversal region can be calculated from equation (7) in the sudden approximation, assuming that the change in phase angle is negligibly small. The normalised transition probability, $|I(r, v)|^{2}$, for atoms with radial coordinate $r$ is approximately obtained from

$$
|I(r, v)|=\int_{t_{0}}^{i_{1}} \omega_{\mathrm{F}}(t) \sin \theta(t) \mathrm{d} t
$$

with

$$
\omega_{\mathrm{F}}(t) \sin \theta(t)=g_{\mathrm{F}}\left(\mu_{\mathrm{B}} / \hbar\right) B_{\mathrm{r}} .
$$

The radial field, $B_{\mathrm{r}}$, is obtained from div $\boldsymbol{B}=0$ as

$$
B_{\mathrm{r}}=B_{\mathrm{M}}(r / d) \text {. }
$$

This leads to the final result:

$$
|I(r, v)|^{2}=[4 \pi r /(L \ln \chi)]^{2}
$$

The transition probability is independent of the length of the reversal region as long as the sudden approximation holds. However, from a practical point of view, it is recommended to keep the reversal region short as the radial magnetic field $B_{\mathrm{r}}$ is then relatively large and the requirements on the control of stray magnetic fields are less stringent.

One concludes from equation (12) that for fixed $\chi$ only the ratio of the radial beam extension $R$ to the total length of the flipper $L$ is the major design parameter (in our case $R=1.5 \mathrm{~mm}$. $L=320 \mathrm{~mm}$ ). Using the flipper for atomic species with different magnetic moments and different beam velocities requires scaling of the magnetic field according to equation (8).

The layout of the spin flipper is shown in figure 2 . The wall of the vacuum chamber is made out of regular nonmagnetic stainless steel (German steel number 1.4571 , equivalent to SS 316 ). The different coils are mounted inside a magnetic shield which is a cylinder made out of $1 \mathrm{~mm}$ Hyperm 760. Different field shapes can be tried out as the currents in six pairs of coils, arranged symmetrically to the midplane, are separately adjustable. In order to ensure that the zero-field point lies exactly on axis two transverse coils, orthogonal to each other (see figure 2), can be used for fine adjustment. The same coils are used for applying a transverse magnetic field of about $0.1 \mathrm{mT}$ at the zero-field point when switching to the adiabatic mode of the spin flipper.

\section{Performance of the spin flipper}

The spin flipper was built for our polarisation experiment on electron-lithium scattering. Part of the experimental set-up was used for testing the spin flipper and is shown in figure 3 . A lithium- 6 atomic beam is polarised by high-field state selection in a sextupole magnet. The polarised atomic beam traverses the spin flipper, the collision chamber, and the analysing sextupole magnet before hitting the hot-wire detector. The performance of the spin flipper can best be studied by using a second spin-flip region, which does not necessarily need to have a high flipping efficiency. As it turned out, the magnetically shielded collision chamber can easily be used for that purpose. Only minor experimental changes were required: the guiding field on one side of the chamber had to have opposite direction from that on the other side. In addition, the Earth's field had to be compensated. Thus there are four operational modes for polarisation transport, leading to different atomic beam signals at the hot-wire detector, namely, $I^{\text {no }}$ (no flip at all), $I^{\mathrm{F}}$ (spin flipper acting alone), $I^{F^{\prime}}$ (second spin-flip region acting alone), and $I^{\mathrm{FF}^{\prime}}$ (one flip in each region).

In order to determine the transition probability from the observed hot-wire signals some properties of the sextupole 


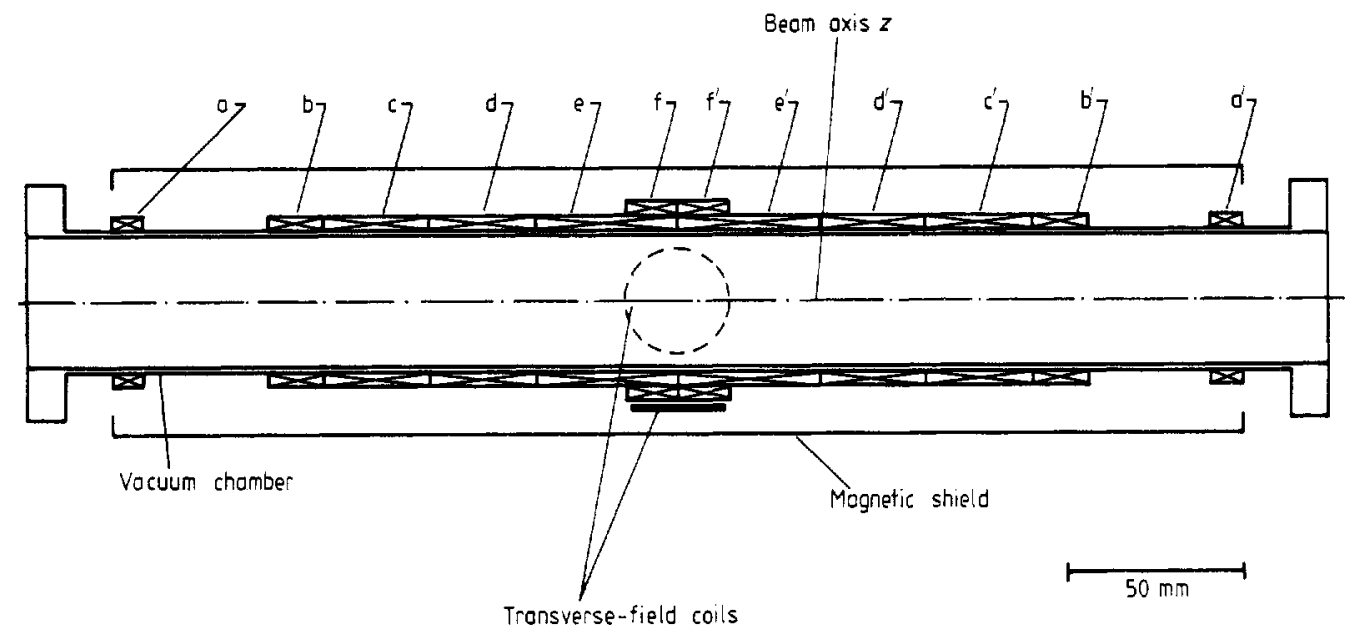

Figure 2. Cross section of the spin flipper. The longitudinal coils, connected in pairs, have the following values for number of turns and current (which is given in parentheses): coil a (likewise coil a), 22(600 mA); coil b, 18(14 mA); coil c, 60(5 mA); coil d, 28(5 mA); coil e, $27(5 \mathrm{~mA})$; coil f, $23(7 \mathrm{~mA})$. The transverse field coils (10 turns) are needed for adjusting the location of the zero-field point and carry an additional current of $300 \mathrm{~mA}$ in the adiabatic mode.

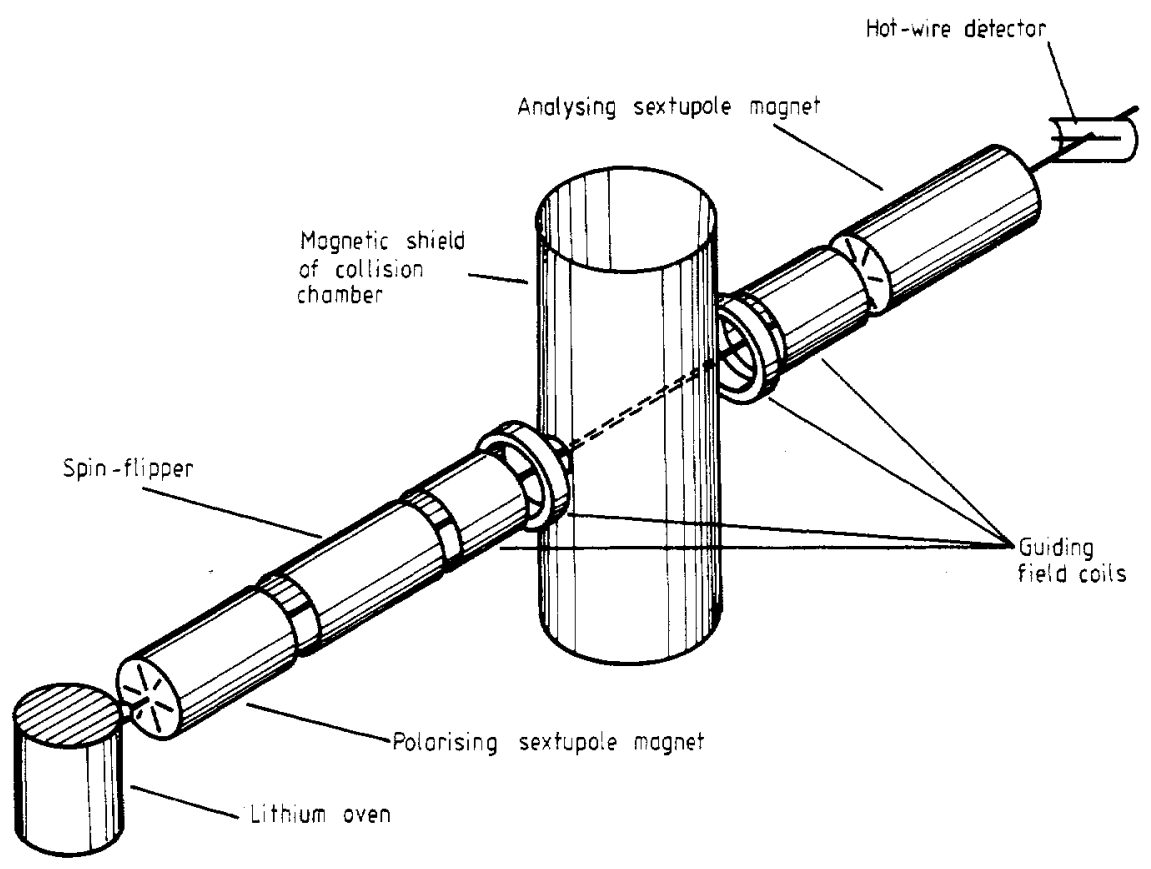

Figure 3. Assembly drawing of the atomic beam system. For using the collision chamber as second spin-flip region, the downstream magnetic guiding field is reversed, opposing the upstream guiding field.

magnets have to be known. A sextupole magnet transmits atoms in the high-field states with $m_{5}=+1 / 2, m_{1}=-1,0,+1$ (these connect to the low-field, hyperfine-coupled states $F=3 / 2$, $m_{\mathrm{F}}=-1 / 2,+1 / 2,+3 / 2$ ). Atoms in the states with $m_{\mathrm{s}}=-1 / 2$ (connecting to $F=3 / 2, m_{\mathrm{F}}=-3 / 2 ; F=1 / 2, m_{\mathrm{F}}=-1 / 2,+1 / 2$ ) are defocused. Our polarising magnet (gap radius $1.6 \mathrm{~mm}$, poletip field $0.87 \mathrm{~T}$ ) has a length of $76 \mathrm{~mm}$. It is not strong enough to provide a complete high-field state selection. A small fraction of the atoms leaving the magnet are in states with $m_{\mathrm{s}}=-1 / 2$. The effect of the spin-flip regions on the state populations of the atoms is analysed with an additional sextupole magnet. The analysing magnet (gap radius $1.6 \mathrm{~mm}$, pole-tip field $0.87 \mathrm{~T}$ ) has a length of $234 \mathrm{~mm}$ and provides perfect high-field state selection. It transmits only atoms in states with $m_{s}=+1 / 2$ to the hot-wire detector and rejects all atoms in states with $m_{\mathrm{s}}=-1 / 2$.

The probability $\eta$ for transitions from $F=3 / 2, m_{\mathrm{F}}=-1 / 2$ to $m_{\mathrm{F}}=-3 / 2$ under the combined action of spin flipper and second spin-flip region can be calculated from the hot-wire signals as

$$
\eta=\eta^{F}+\eta^{\mathrm{F}^{\prime}}=3\left(I^{\mathrm{no}}-I^{\mathrm{FF}}\right) / I^{\mathrm{no}}
$$

For low transition rates depolarising transitions are of concern only between those adjacent states, where one state is highly populated and the other one nearly empty. For the level with $F=3 / 2$ this applies to one third of the state-selected atoms, explaining the factor 3 in equation (13). For $\eta$ we measured a value of $\eta=0.044$, which constitutes an upper limit for the 
transition probability $\eta^{\mathrm{F}}$ of the spin flipper. More information on $\eta^{F}$ is obtained by studying the variation of $\eta$ with an intentional dislocation of the zero-field point from the atomic beam axis. For this purpose the compensating currents in the transverse coils of the spin flipper are misadjusted, changing $\eta^{\mathrm{F}}$ but leaving $\eta^{F^{\prime}}$ constant. These measurements are shown in figure 5 . One obtains a value of $\eta^{\mathrm{F}}=0.034$ for an optimally tuned spin flipper (see equation (17)). The efficiency of the spin flipper, $\varepsilon$, is calculated to be

$$
\varepsilon \equiv P_{\mathrm{a}}^{\mathrm{F}} / P_{\mathrm{a}}^{\mathrm{no}}=1-2 / 3 \eta^{\mathrm{F}}=0.98
$$

where $P_{\mathrm{a}}^{\mathrm{F}}$ and $P_{\mathrm{a}}^{\mathrm{no}}$ denote the atomic beam polarisations for the reversing (diabatic) and nonreversing (adiabatic) mode, respectively.

The experimental studies showed that $\varepsilon$ does not critically depend on the currents in the coils of the spin fipper. The current through coil $b$ should lie below $30 \mathrm{~mA}$. The current through coils $\mathrm{c}, \mathrm{d}$, e should lie between $20 \mathrm{~mA}$ and $3 \mathrm{~mA}$. The current through coil $f$ has the greatest influence on $\varepsilon$. The stray fields have to be compensated by means of the transverse field coils until they lie below $0.1 \mu \mathrm{T}$.

As mentioned above, our polarising sextupole magnet produces only an incomplete high-field state selection. The resulting low-field electronic polarisation $P_{\mathrm{a}}$ can be determined very accurately by utilising the spin flipper together with the analysing sextupole magnet. For this purpose we compare the hot-wire signals for the spin flipper operating in the adiabatic mode $\left(I^{\mathrm{no}}\right)$ and in the diabatic mode $\left(I^{\mathrm{F}}\right)$. Under the assumption of equal population within the three states with $m_{\mathrm{s}}=+1 / 2$ and equal population within the three states with $m_{s}=-1 / 2$ the atomic polarisation can be calculated from

$$
P_{\mathrm{a}}=\left(1-I^{\mathrm{F}} / I^{\mathrm{no}}\right) /\left(3 I^{\mathrm{F}} / I^{\mathrm{no}}-1\right) \text {. }
$$

The measured value of $P_{\mathrm{a}}=0.27$ is in agreement with expectations based on trajectory calculations for a mean thermal velocity of $v=2000 \mathrm{~m} \mathrm{~s}^{-1}$. A complete high-field state selection would yield $P_{\mathrm{a}}=0.33$ for ${ }^{6} \mathrm{Li}$.

\section{Comparison with calculations}

A computer program was used to calculate the magnetic field on axis for a given current configuration, to calculate the radial field component from the relation $\operatorname{div} \boldsymbol{B}=0$, and to calculate the transition probability from equation (7). For evaluating the magnetic fields the step size was $10 \mu \mathrm{m}$. Along the path of the atom $I(r, v)$ was integrated in phase steps of $0.01 \mathrm{rad}$ for different values of $r$ and $v$, and for different starting points in the entrance region of the spin flipper. The calculations show that the probability for depolarising transitions in the adiabatic transport regions is very small, as can be seen in figure 4 . In the transport regions small oscillations occur because $\mathrm{d} B / \mathrm{d} z$ is nonzero. In the exit region the phase of these oscillations is independent of the starting point, contrary to the behaviour in the entrance region. This feature, however, is irrelevant for the flipper performance, as an averaging over all starting phases occurs.

In the diabatic mode the main contribution to the depolarising transition probability comes from the central region. For calculating the phase-averaged transition probability $p(r, v)$ as a function of $r$ and $v$, we performed several $I(r, v)$ computations with different starting points along the $z$ axis (results for two of these are shown in figure 4) and averaged over all starting points. For the investigated velocity range of 600 to $5000 \mathrm{~m} \mathrm{~s}^{-1}$ it was found that $p(r, v)$ is proportional to $1 / v$ and depends on $r$ in the following way:

$$
p(r, v)=\alpha(v) r^{2}
$$

This relation is in agreement with the model of Hight et al

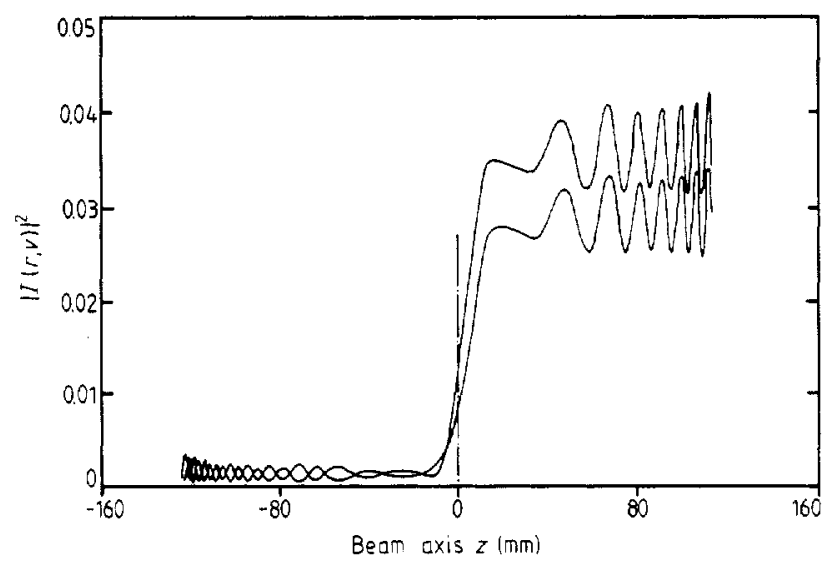

Figure 4. Normalised transition probability $|I(r, v)|^{2}$ of equation (6) along the fiipper axis for two different starting points and for $r=1 \mathrm{~mm}, v=2000 \mathrm{~m} \mathrm{~s}^{-1}$.

(1977). If $\alpha(v)$ is small, large beam diameters can be tolerated for a well-working flipper. For $v=2000 \mathrm{~m} \mathrm{~s}^{-1}$ and an off-axis parameter $r=1 \mathrm{~mm}$ we find that $p(r, v)$ equals 0.03 .

In order to make a comparison with the measurements, further averaging is necessary. The transition probability $p(r, v)$ is folded with the atomic beam velocity distribution, and averaged over the atomic beam profile in the flip region. A possible radial offset, $a$, of the zero-field point from the beam

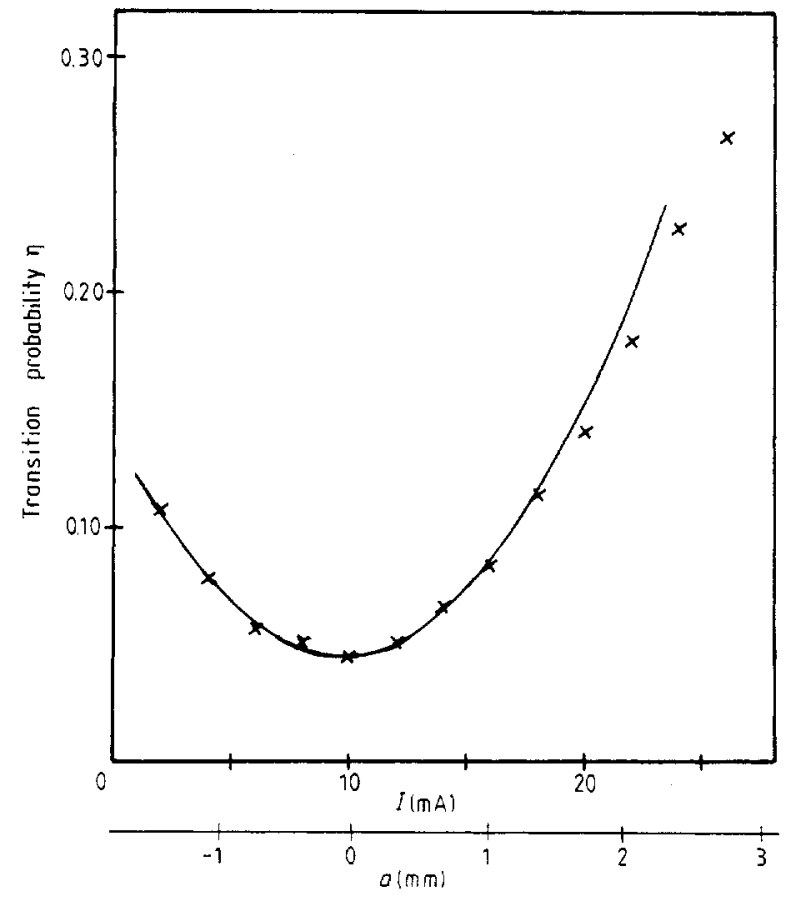

Figure 5. Measured transition probability $\eta$ as function of transverse coil current $I . \times$, data points. The full curve is a parabola fitted to the data points near the minimum. The displacement of the zero-field point from axis, a, corresponding to a certain coil current $I$, is calculated from the geometry of the transverse field coil. 
axis has to be taken into account by applying the parallel-axis theorem:

$\eta^{F}(a)=\bar{\alpha}\left[a^{2}+\left(\int_{0}^{R} \rho(r) r^{2} 2 \pi r \mathrm{~d} r\right) /\left(\int_{0}^{R} \rho(r) 2 \pi r \mathrm{~d} r\right)\right]$.

The average of $\alpha(v)$ over the velocity distribution is denoted by $\bar{\alpha}$. The beam radius in the flip region is $R=1.5 \mathrm{~mm}$. The measurements, shown in figure 5, are fitted by a parabola and, using equation (17), a value of $\bar{\alpha}=0.03 \pm 0.01 \mathrm{~mm}^{-2}$ is extracted. This value agrees well with the results of our calculations. The deviation of the data points from the parabola at high transition probabilities is expected from the approximation used in the model.

\section{Summary}

For reversing the direction of polarisation in a lithium atomic beam an efficient spin flipper was built. The design criteria given can be easily adapted for other spin-polarisable atoms. For adjusting the spin flipper and measuring its performance, a second spin-flip region and an analysing sextupole magnet have been utilised. The experimental set-up also allowed a determination of the atomic beam polarisation.

\section{Acknowledgments}

We are grateful to $U$ Sillmen who assisted during the measurements. We wish to thank Professor W Raith for many valuable discussions. The work was supported by the University of Bielefeld under Project No 2845.

\section{References}

Baum G, Kisker E, Raith W, Schoeder W, Sillmen U and

Zenses D 1981 Spin-dependent electron impact ionisation of lithium from threshold to $80 \mathrm{eV}$

J. Phys. B: At. Mol. Phys. 14 4377-88

Donally B L 1970 Lamb-shift source Proc. 3rd Int. Symp.

on Polarisation Phenomena in Nuclear Reactions

ed. $\mathrm{H} \mathrm{H}$ Barschall and $\mathrm{W}$ Haeberli (Madison: Univ. Wisconsin Press 1971) 295-316

Frisch R and Segré E 1933 Über die Einstellung der

Richtungsquantelung. II

Z. Phys. $80610-6$

Hight R D, Robiscoe R T and Thorson W R 1977 Nonadiabatic spin transitions in an inhomogenous magnetic field

Phys. Rev. A 15 1079-87

Hight R D and Robiscoe R T 1978 Nonadiabatic transitions in $n=2$ atomic hydrogen

Phys. Rev. A 17 561-5

Liaud P, Steinberg R I and Vignon B 1975 A two-coil spin-

flipper for beams of polarized slow neutrons

Nucl. Instrum. Meth. 125 7-8

Majorana E 1932 Atomi orientati in campo magnetico variable Nuovo Cimento 943

McKibben J L 1981 Survey of methods for rapid spin reversal Proc. 5th Int. Symp. on Polarization Phenomena in Nuclear Physics, 1980 ed. G G Ohlsen et al

(AIP Conf. Proc. No. 69, New York) pp 830-47 